\title{
Frequent nasopharyngeal suctioning as a risk factor associated with neonatal coagulase-negative staphylococcal colonisation and sepsis
}

\author{
Nem Yun $\underline{B o o}^{1}$, MBBS, FRCPCH, Abdul Rahman $\underline{\text { Suhaida }}^{2}$, MMed, Jaafar $\underline{\text { Rohana }}^{2}$, MMed, FAMM
}

INTRODUCTION This case-control study aimed to determine whether catheter use was significantly associated with coagulase-negative staphylococci (CoNS) colonisation and/or sepsis in neonates.

METHODS Weekly swabs of the nose, umbilicus, rectum, wounds, eye discharge and intravenous catheter tips (after removal) of infants admitted to the neonatal intensive care unit of Universiti Kebangsaan Malaysia Medical Centre, Malaysia, were cultured. CoNS sepsis was diagnosed if pure growth of CoNS was cultured from the peripheral blood specimen of symptomatic infants. For each infant with CoNS colonisation or sepsis, a control infant was retrospectively and randomly selected from unaffected infants in the ward. Multivariate analyses were performed to determine whether catheter use was a significant risk factor.

RESULTS CoNS colonisation was detected in 113 (8.7\%) infants. CoNS sepsis was found in 12 (10.6\%) infants with CoNS colonisation and $7(0.6 \%)$ infants without CoNS colonisation. Multivariate analysis showed that the following were significantly associated with CoNS colonisation: conjunctivitis (adjusted odds ratio [OR] 8.2, 95\% confidence interval [CI] 1.9-34.8, $\mathrm{p}=0.005$ ); central venous catheters (adjusted OR 5.8, 95\% Cl 1.9-17.8, $\mathrm{p}=0.002$ ); and nasopharyngeal and/or oral suctioning more than twice in the 48 hours before positive culture (adjusted OR 7.3, 95\% $\mathrm{Cl} 3.3-16.2, \mathrm{p}<0.001$ ). Exposure to frequent nasopharyngeal and/or oral suctioning (adjusted OR 20.8, 95\% Cl 3.5-125.3, p = 0.001) was the only significant factor associated with CoNS sepsis.

CONCLUSION Infants requiring more than two nasopharyngeal and/or oral suctions in the previous 48 hours were found to have a higher risk of developing CoNS colonisation and sepsis.

Keywords: colonisation, CoNS, neonates, NICU, sepsis

\section{INTRODUCTION}

Coagulase-negative staphylococci (CoNS) are part of the normal flora of the skin and mucosa. When the integrity of the skin or mucosa is breached (e.g. by central venous catheters [CVCs] or oral gastric tubes [OGTs]), CoNS can gain entry into the host. ${ }^{(1)}$ The biofilm-forming capacity of CoNS was thought to be the main virulence factor protecting embedded CoNS against the host's response mechanisms and antibiotics. ${ }^{(2)}$

In the last 20 years, CoNS has been reported to be a common cause of sepsis in many neonatal intensive care units (NICUs). ${ }^{(3-8)}$ Molecular studies in the recent years suggest that mucosal sites could be more important sources of CoNS sepsis than the skin, in both adults and children..$^{(9)}$ Although studies have reported various risk factors associated with CoNS sepsis in NICUs, ${ }^{(2,10,11)}$ only a few small series have reported a possible association between mucosal colonisation and CoNS sepsis. ${ }^{(12-14)}$ The present study aimed to determine whether the use of catheters in neonates via various routes (e.g. CVCs, nasopharyngeal catheters, OGTs and intravenous catheters) was significantly associated with CoNS colonisation and/or sepsis.

\section{METHODS}

This case-control study was carried out in the NICU of Universiti Kebangsaan Malaysia Medical Centre, Malaysia, over a 20-month period (1 February 2008 to 30 September 2009). The hospital's scientific and ethics committees approved the study's protocol. Parental consent was obtained for the inclusion of the infants in the study. All infants admitted to the NICU during the study period were eligible for inclusion. Routine baseline nasal, umbilical and rectal swabs for microbiological culture were obtained from each infant on admission and weekly until the time of discharge. Nasopharyngeal aspirates for microbiological culture were obtained weekly from infants on ventilation and nasal continuous positive airway pressure (nCPAP) therapy. The tips of all catheter devices (e.g. endotracheal tubes, umbilical venous/arterial catheters and percutaneously inserted central catheters) were sent for microbiological culture upon removal. Surface swabs were taken for microbiological culture from infants with eye discharge, surgical wounds, and/or omphalitis (i.e. foul-smelling discharge and/or redness at the base of the umbilical cord). If an infant developed signs of sepsis (e.g. fever, hypothermia, lethargy and feed intolerance), $0.5-1.0 \mathrm{~mL}$ of blood was drawn via sterile technique from the infant's peripheral vein (after skin cleansing with Hibitane in spirit [1 in 200] for at least $30 \mathrm{~s}$ ) and sent for microbiological culture. A diagnosis of CoNS sepsis was made if pure growth of CoNS was cultured from the blood sample of a symptomatic infant. An infant was diagnosed with CoNS colonisation if

${ }^{1}$ Faculty of Medicine and Health Sciences, Universiti Tunku Abdul Rahman, Selangor, ${ }^{2}$ Neonatal Intensive Care Unit, Universiti Kebangsaan Malaysia Medical Centre, Kuala Lumpur, Malaysia Correspondence: Dr Boo Nem-Yun, Emeritus Professor, Faculty of Medicine and Health Sciences, Universiti Tunku Abdul Rahman, Bandar Sungai Long, 43000 Kajang, Selangor, Malaysia. boony@utar.edu.my 
CoNS were isolated from any of the aforementioned swabs or catheter tips.

A 'case' was defined as an infant confirmed to have CoNS sepsis and/or colonisation. For each case, one control infant was recruited. To minimise bias due to changes in nursing practices during the study period and errors due to the recruitment of infants who might later be defined as a case, each control infant was retrospectively selected (at or after discharge) among unaffected infants who stayed in the ward on the same day that a case was detected. A random number table was used to select the control infants. We did not match each case with a control of similar gestation and birth weight, as these two factors were among the risk factors examined. The reference point for the timing of various procedures (e.g. OGT in situ, nasopharyngeal suction in the previous 48 hours) in a control infant was matched with the day its corresponding case infant was diagnosed. Data on the clinical problems and outcomes of all the infants recruited were obtained from their respective observation charts and patient records, and entered into a standard format for analysis.

In the present study, the practice of routine nasopharyngeal and/or oral suctioning of infants is not encouraged in the NICU. This is to minimise the risk of mucosa trauma and sepsis. However, nasopharyngeal and/or oral suctioning would be carried out by nurses if the infants developed severe respiratory distress or desaturation due to excessive secretion. Any suctioning performed was recorded in the observation chart. In the present study, nasopharyngeal suctioning was defined to be frequent if it was carried out more than two times in the previous 48 hours.

Blood cultures were performed with the BACTEC 9240 system using paediatric bottles (Becton, Dickinson and Company, Meylan, France). The culture bottles were incubated at $37^{\circ} \mathrm{C}$ for a maximum of seven days, except when positive culture occurred at an earlier time. Identification of the microorganisms in the culture was based on the results of Gram-staining and the examination of colony-forming units in blood agar and tryptic soy agar plates. All nasal, umbilical, skin, eye discharge and wound swabs collected were plated onto blood agar prepared in our own laboratory, and incubated at $37^{\circ} \mathrm{C}$ for 48 hours. Positive isolates were similarly identified.

Based on a $40 \%$ incidence rate of CoNS sepsis among infants with CoNS colonisation in the same NICU in 2007, a minimum sample size of 100 colonised infants must be recruited for the present study in order to detect an incidence of CoNS sepsis among colonised infants with a relative precision of $50 \%$ of the true value and with a power of $90 \%$.

Statistical analyses were performed using the Statistical Package for the Social Sciences version 18.0 (SPSS Inc, Chicago, IL, USA). The demographic data, clinical problems and catheter use of the cases and controls were compared. Chi-square test or Fisher's exact test (for expected value $<5$ ) was used for the analysis of categorical variables. Student's $t$-test was used for the analysis of numerical variables with a normal distribution, while Mann-Whitney $U$ test was used for the analysis of numerical variables with a skewed distribution.
If multiple episodes of CoNS sepsis occurred in the same infant, only the first episode was included in the analysis. Forward logistic regression analysis was carried out to identify significant risk factors associated with CoNS colonisation; the independent variables were birth weight, gestational age, conjunctivitis, blood sampling practice, CVC in situ, total parenteral nutrition (TPN), OGT in situ, nCPAP therapy, conventional ventilation therapy, and nasopharyngeal and/or oral suctioning of more than twice in the previous 48 hours. Similarly, forward logistic regression analysis was carried out with CoNS sepsis as the dependent variable and the following as independent variables: duration of TPN; duration of nCPAP; nasopharyngeal and/or oral suctioning of more than twice in the previous 48 hours; OGT in situ in the previous 48 hours; and blood sampling in the previous 48 hours. A p-value $<0.05$ was considered statistically significant.

\section{RESULTS}

During the 20-month period, 1,293 infants were admitted to the NICU of Universiti Kebangsaan Malaysia Medical Centre, Malaysia. CoNS colonisation was detected in 113 (8.7\%) infants at a median age of 8 (interquartile range [IQR] 4,13) days. Of these 113 infants, $12(10.6 \%)$ developed CoNS sepsis. Seven other infants without evidence of CoNS colonisation also developed sepsis (Fig. 1). Thus, a total of 19 infants developed CoNS sepsis, giving an incidence of 1.5 per 100 admissions. The median age of onset of CoNS sepsis was 12 (IQR 6, 22; range 1-61) days. The most common sites of CoNS colonisation were the nasopharyx $(n=47)$, endotracheal tube $(n=23)$ and eyes $(n=18)$ (Fig. 2). CoNS organisms were detected at more than one site in $21(18.6 \%)$ infants. None of the infants had CoNS cultured from their umbilical or rectal swabs. Only one infant had a nasal swab positive for CoNS. In the 12 infants with both CoNS colonisation and sepsis, CoNS was cultured from the tip of endotracheal tubes $(n=6)$, the nasopharynx $(n=5)$ and the tip of an umbilical venous catheter $(n=1)$.

Of the 120 infants with CoNS colonisation and/or sepsis (Fig. 1), 101 infants had 1 control infant recruited for each of them. No control infants were recruited for the remaining 19 infants (18 cases of CoNS colonisation and 1 case of CoNS sepsis), as the lists of infants hospitalised in the NICU on the days that CoNS colonisation and/or sepsis occurred in these 19 infants were not available for random selection of controls. There was no significant difference in the ethnic $(p=0.4)$ or gender distribution $(p=0.5)$, median birth weight $(p=0.5)$, gestational age $(p=0.4)$ and age at colonisation $(p=0.8)$ between the infants with and without controls.

The data presented in the following paragraph is that of the 101 cases and their controls. When compared with the controls, the infants with CoNS colonisation had significantly lower birth weight and gestational age (Table I). Between the controls and the cases, there were no significant differences in gender and ethnic distribution, the number of infants who were delivered via lower segment Caesarean section, the age at admission, the number of infants with surgical wounds, the number of infants with omphalitis, the type of milk received, the number of infants handled by their parents in the previous 24 hours, and the number of infants who had kangaroo care in the previous 24 hours. 


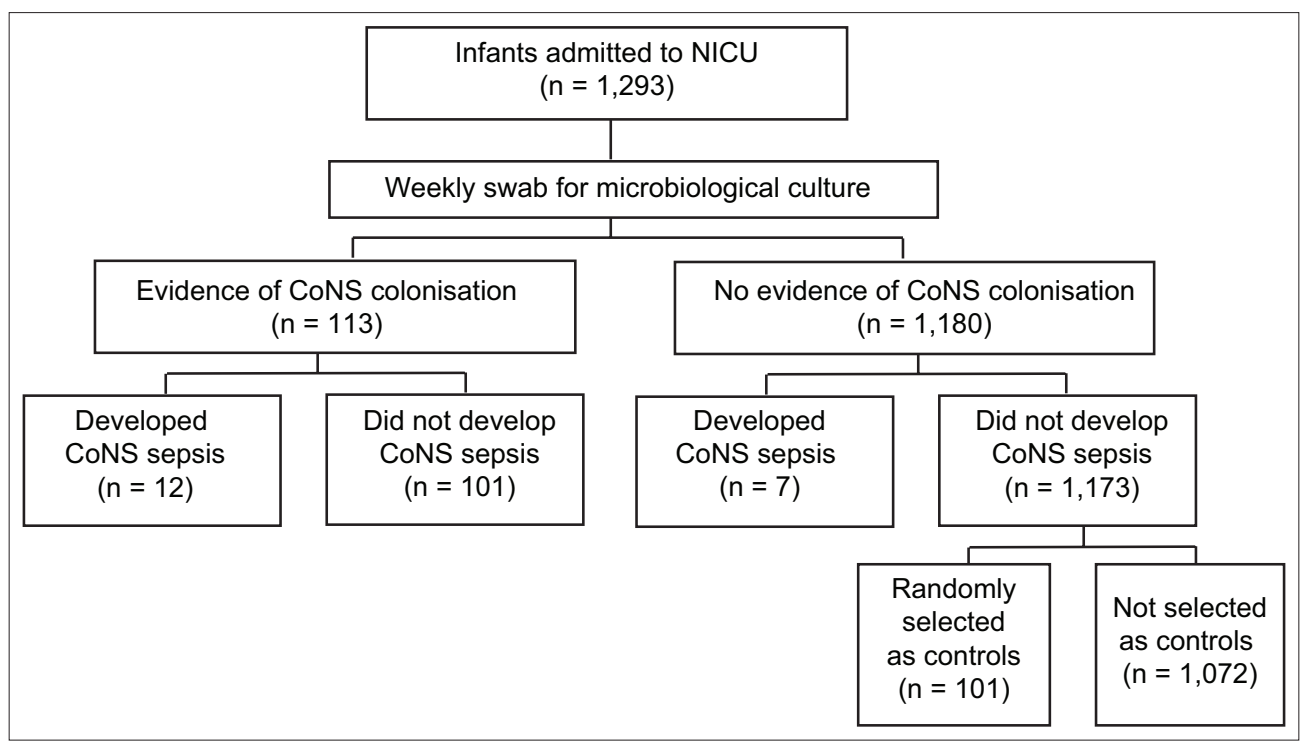

Fig. 1 Flowchart shows the enrolment process and the outcomes of infants admitted to the neonatal intensive care unit (NICU).

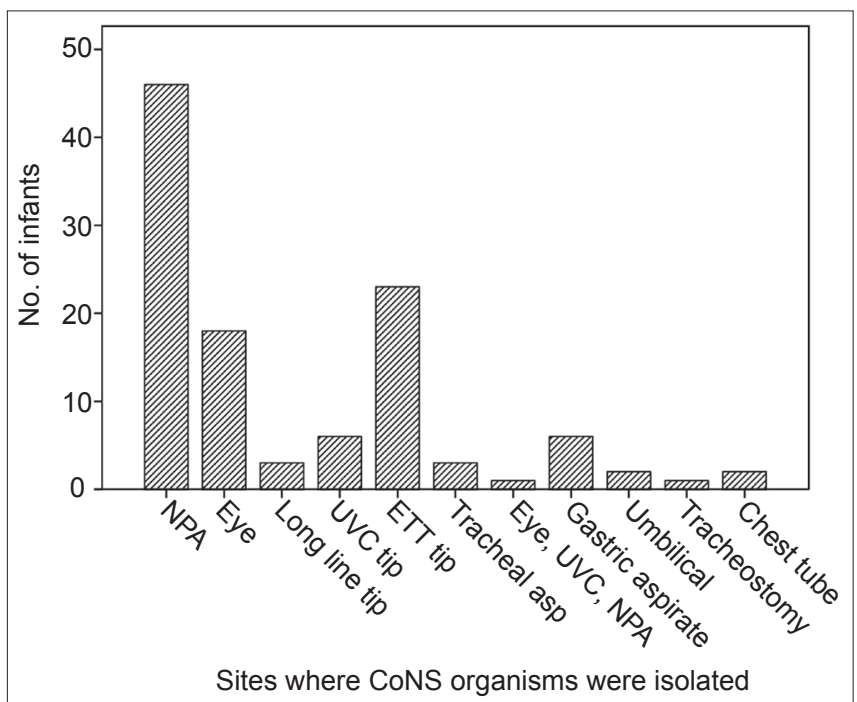

Fig. 2 Bar chart shows the sites where coagulase-negative staphylococc (CoNS) were isolated. CVC: central venous catheter; ETT: endotracheal tube; NPA: nasopharynx aspirate; UVC: umbilical venous catheter

A significantly higher proportion of infants who had CoNS colonisation were nursed in the intensive care area, had a history of eye discharge in the previous week, and had blood sampling, CVC in situ, TPN, and OGT in situ in the previous 48 hours. Infants with CoNS colonisation also received a significantly longer duration of TPN prior to the date of CoNS colonisation. A significantly higher proportion of infants with CoNS colonisation were on nCPAP therapy, conventional ventilatory support, and frequent nasopharyngeal and/or oral suctioning in the previous 48 hours. Very few infants in both the case and control groups had continuous bladder drainage in the previous 48 hours.

Logistic regression analysis showed that the significant risk factors associated with CoNS colonisation were: (a) eye discharge in the previous 48 hours (adjusted odds ratio [OR] 8.2, 95\% confidence interval [Cl] 1.9-34.8, $p=0.005$ ); (b) CVC in situ (adjusted OR 5.8, 95\% Cl 1.9-17.8, $\mathrm{p}=0.002$ ); and (c) nasopharyngeal and/or oral suctioning on more than two occasions in the previous 48 hours (adjusted OR 7.3, 95\% Cl 3.3-16.2, $\mathrm{p}<0.001$ ). Birth weight, gestation, blood sampling, TPN, nCPAP, conventional ventilation, and OGT in situ in the previous 48 hours were not significant risk factors ( $p>0.05$ ).

When compared with the controls, a significantly higher proportion of infants with CoNS sepsis were nursed in the intensive care area on the day of sepsis, and had blood sampling, CVC in situ, TPN, OGT in situ and nCPAP therapy in the previous 48 hours. Infants with CoNS sepsis received a significantly longer duration of TPN and nCPAP therapy prior to CoNS sepsis. A significantly higher proportion of these infants also had more than two nasopharygeal and/or oral suctioning procedures performed on them in the previous 48 hours. Although there was no significant difference in the mortality rates between the case and control groups, infants with CoNS sepsis had a significantly longer duration of hospital stay.

Logistic regression analysis showed that the only significant risk factor associated with CoNS sepsis was a history of having received nasopharyngeal and/or oral suctioning on more than two occasions in the previous 48 hours (adjusted OR 20.8, 95\% CI 3.5-125.3, $p=0.001$ ). Duration of CVC in situ, TPN and nCPAP, a history of OGT in situ, and blood samplings in the previous 48 hours were not significant risk factors ( $p>0.05)$.

When infants with CoNS colonisation and no sepsis $(n=101)$ were compared with infants with CoNS colonisation and sepsis $(\mathrm{n}=12)$, univariate analysis showed that a significantly higher proportion of infants with CoNS colonisation and sepsis were on intravenous drip (100.0\% vs. 61.9\%, p =0.008), had CVC in situ in the previous 48 hours $(91.7 \%$ vs. $35.0 \%, p<0.001)$, had TPN in the previous 48 hours $(91.7 \%$ vs. $30.3 \%, \mathrm{p}<0.001)$, had a longer duration of TPN ( 9 days vs. 2 days, $\mathrm{p}<0.0001$ ), were not on full enteral feeds (83.3\% vs. $53.5 \%, p=0.049)$, and had a higher mortality rate $(25.0 \%$ vs. $4.0 \%, p=0.03)$.

\section{DISCUSSION}

In the present study, CoNS colonisation was high and $10.6 \%$ of the infants who had CoNS colonisation developed sepsis. We found 
Table I. Comparison of the demographic and clinical variables of infants with coagulase-negative staphylococci (CoNS) colonisation or CONS sepsis, and their controls.

\begin{tabular}{|c|c|c|c|c|c|c|}
\hline \multirow[t]{3}{*}{ Variables } & \multicolumn{6}{|c|}{ No. (\%) } \\
\hline & \multicolumn{3}{|c|}{ CoNS colonisation } & \multicolumn{3}{|c|}{ CoNS sepsis } \\
\hline & Cases $(n=95)$ & Controls $(n=95)$ & p-value & Cases $(n=18)$ & Controls $(n=18)$ & p-value \\
\hline Birth weight* (g) & $1,820 \pm 889$ & $2,224 \pm 780$ & $0.001^{*}$ & $1,248(861,3,280)$ & $2,650(1,618,3,364)$ & 0.5 \\
\hline Gestation* (wk) & $32.7 \pm 4.4$ & $34.7 \pm 3.3$ & $<0.0001^{*}$ & $30.5(27.8,38.5)$ & $37.0(33.5,39.3)$ & 0.7 \\
\hline Male gender & $56(58.9)$ & $52(54.7)$ & 0.3 & $10(55.6)$ & $8(44.4)$ & 0.5 \\
\hline Delivered by LSCS & $52(54.7)$ & $45(47.4)$ & 0.7 & $9(50.0)$ & $7(38.9)$ & 0.2 \\
\hline Age at admission ${ }^{+}$(day) & $1(1,1)$ & $1(1,1)$ & 0.3 & $1.0(1.0,1.8)$ & $1.0(1.0,3.3)$ & 0.7 \\
\hline $\begin{array}{l}\text { Environment where infants were nursed } \\
\text { when CoNS colonisation/sepsis was } \\
\text { detected in the cases }\end{array}$ & & & $<0.0001^{*}$ & & & $0.002^{\ddagger}$ \\
\hline Intensive care area & $63(66.3)$ & $22(23.2)$ & & $12(66.7)$ & $1(5.6)$ & \\
\hline Intermediate care area & $11(11.6)$ & 36 (37.9) & & $3(16.7)$ & $11(61.1)$ & \\
\hline Isolation area & $11(11.6)$ & $7(7.4)$ & & $1(5.6)$ & $2(11.1)$ & \\
\hline Convalescent care area & $10(10.5)$ & $30(31.6)$ & & $2(11.1)$ & $4(22.2)$ & \\
\hline Had surgical wound in the previous week & $15(15.8)$ & $10(10.5)$ & 0.3 & $6(33.3)$ & $3(16.7)$ & 0.4 \\
\hline Had omphalitis in the previous week & $2(2.1)$ & $5(5.3)$ & 0.3 & $0(0)$ & $1(5.6)$ & 1.0 \\
\hline Had conjunctivitis in the previous week & $12(12.6)$ & $4(4.2)$ & $0.04^{*}$ & $0(0)$ & $1(5.6)$ & 1.0 \\
\hline On intravenous drip in the previous $48 \mathrm{hr}$ & $62(65.3)$ & $56(58.9)$ & 0.3 & $16(88.9)$ & $11(61.1)$ & 0.1 \\
\hline $\begin{array}{l}\text { Had blood sampling performed in the } \\
\text { previous } 48 \mathrm{hr}\end{array}$ & $42(44.2)$ & $12(12.6)$ & $<0.0001^{\ddagger}$ & $10(55.6)$ & $4(22.2)$ & $0.04^{*}$ \\
\hline Had CVC in situ in the previous $48 \mathrm{hr}$ & $40(42.1)$ & $7(7.4)$ & $<0.0001^{*}$ & $11(61.1)$ & $3(16.7)$ & $0.006^{\ddagger}$ \\
\hline On TPN during the previous $48 \mathrm{hr}$ & $36(37.8)$ & $5(5.3)$ & $<0.0001^{*}$ & $12(66.7)$ & $2(11.1)$ & $0.001^{*}$ \\
\hline $\begin{array}{l}\text { Duration of TPN received when CoNS } \\
\text { colonisation/sepsis was detected in the } \\
\text { cases }^{+} \text {(day) }\end{array}$ & $0(0,4)$ & $0(0,0)$ & $<0.0001^{*}$ & $4(0,8)$ & $0(0,0)$ & $0.001^{*}$ \\
\hline Had OGT in situ in the previous $48 \mathrm{hr}$ & $82(86.3)$ & $56(58.9)$ & $<0.0001^{\ddagger}$ & $14(77.8)$ & $8(44.4)$ & $0.04^{*}$ \\
\hline $\begin{array}{l}\text { Type of milk received when CoNS } \\
\text { colonisation/sepsis was detected } \\
\text { in the cases }\end{array}$ & & & 0.3 & & & 0.1 \\
\hline Formula only & $57(60.0)$ & $67(70.5)$ & & $9(50.0)$ & $13(72.2)$ & \\
\hline EBM only & $6(6.3)$ & $2(2.1)$ & & $1(5.6)$ & $0(0)$ & \\
\hline Mixed feeding of EBM and formula & $17(17.9)$ & $18(18.9)$ & & $2(11.1)$ & $4(22.2)$ & \\
\hline Nil orally & $13(13.7)$ & $8(8.4)$ & & $6(33.3)$ & $1(5.6)$ & \\
\hline $\begin{array}{l}\text { On full enteral feeds before CoNS } \\
\text { colonisation/sepsis was detected } \\
\text { in the cases }\end{array}$ & $41(43.2)$ & $67(70.5)$ & $0.001^{*}$ & $5(27.8)$ & $15(83.3)$ & $0.001^{*}$ \\
\hline On nCPAP during the previous $48 \mathrm{hr}$ & $51(53.7)$ & $20(21.1)$ & $<0.0001^{*}$ & $8(44.4)$ & $2(11.1)$ & $0.03^{*}$ \\
\hline $\begin{array}{l}\text { Duration of nCPAP before CoNS } \\
\text { colonisation/sepsis was detected in the } \\
\text { cases }^{+} \text {(day) }\end{array}$ & $1(0,9)$ & $0(0,0)$ & $<0.0001^{\ddagger}$ & $0(0,9)$ & $0(0,0)$ & $0.03^{*}$ \\
\hline $\begin{array}{l}\text { On conventional ventilation during the } \\
\text { previous } 48 \mathrm{hr}\end{array}$ & $25(26.3)$ & $6(6.3)$ & $<0.0001^{*}$ & $5(27.8)$ & $1(5.6)$ & 0.09 \\
\hline On HFOV during the previous $48 \mathrm{hr}$ & $4(4.2)$ & $0(0)$ & 0.1 & $0(0)$ & $0(0)$ & - \\
\hline $\begin{array}{l}\text { Had > } 2 \text { times nasopharyngeal or oral } \\
\text { suctioning in the previous } 48 \mathrm{hr}\end{array}$ & $64(67.4)$ & $17(17.9)$ & $<0.0001^{*}$ & $13(72.2)$ & $2(11.1)$ & $<0.0001^{*}$ \\
\hline $\begin{array}{l}\text { Had continuous bladder drainage in the } \\
\text { previous } 48 \mathrm{hr}\end{array}$ & $0(0)$ & $1(1.1)$ & 0.5 & $0(0)$ & $0(0)$ & - \\
\hline Handled by parent in the previous $24 \mathrm{hr}$ & $62(65.3)$ & $66(69.5)$ & 0.3 & $11(61.1)$ & $13(72.2)$ & 0.6 \\
\hline $\begin{array}{l}\text { Had parental kangaroo care in the } \\
\text { previous } 24 \mathrm{hr}\end{array}$ & $9(9.5)$ & $8(8.4)$ & 0.9 & $1(5.6)$ & $1(5.6)$ & 1.0 \\
\hline Died & & & & $3(16.7)$ & $0(0)$ & 0.2 \\
\hline Age at discharge ${ }^{+}$(day) & & & & $30(12,66)$ & $9(6,19)$ & $0.004^{*}$ \\
\hline
\end{tabular}

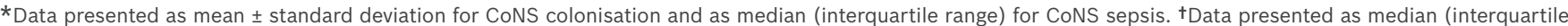
range) for both groups. ¥Data is statistically significant. CVC: central venous catheterisation; EBM: expressed breast milk; HFOV: high frequency oscillation ventilation; LSCS: lower segment Caesarean section; nCPAP: nasal continuous positive airway pressure; OGT: oral gastric tube; TPN: total parenteral nutrition 
that the nasopharynx was the most common site of colonisation, and infants who required more frequent nasopharyngeal and/or oral suctioning had a higher risk of CoNS colonisation and sepsis.

Based on our review of the literature, we believe that the present study is the first to evaluate the association between catheter use and CoNS colonisation and/or sepsis in neonates. Unlike previous studies that focused only on premature/ small infants and vascular catherisation, ${ }^{(2,3,6-8,11,15)}$ the present study included all infants admitted to the NICU of Universiti Kebangsaan Malaysia Medical Centre, Malaysia, and evaluated the effects of exposing the mucosa to catheters. Although univariate analysis showed that premature and small infants were significantly associated with a higher risk of CoNS colonisation, multivariate analysis did not confirm this association.

Similar to the findings of Costa et al, ${ }^{(9)}$ we found that the nasopharygeal mucosa, and not the skin, was the most common site of CoNS colonisation. Similar to Björkqvist et al's findings, ${ }^{(15)}$ the majority of the infants in the present study had CoNS colonisation within the first week of admission. Multivariate analysis showed that other than nasopharyngeal and/or oral suctioning, eye discharge and CVC in situ in the previous 48 hours were also significant risk factors associated with CoNS colonisation in our NICU. These findings suggest that the breach of the skin or mucosa is possibly the most important risk factor associated with CoNS colonisation.

Among the infants with CoNS colonisation in the present study, univariate analysis showed that intravenous drips, CVC and TPN in the previous 48 hours were significant risk factors associated with the development of CoNS sepsis. However, as the number of infants with CoNS colonisation who developed sepsis was small, it was not possible to carry out meaningful multivariate analysis for the identification of significant risk factors associated with sepsis. The small number of infants on expressed breast milk also prevented us from determining whether infants receiving exclusive human milk feeding early in life would have a significantly lower incidence of late-onset CoNS sepsis. ${ }^{(16)}$ We also found that parental handling and kangaroo care did not increase the risk of CoNS colonisation or sepsis. This is an important finding, as it reassures parents that their involvement in the care of their sick infants in the NICU does not contribute to the emergence of CoNS colonisation or sepsis.

A major limitation of the present study was our inability to carry out molecular studies on the relatedness of the organisms isolated from the mucosa and blood of the affected infants. A second limitation was the oversight of collecting data on the infants' underlying clinical problems such as increased airway secretion and compromised ventilation, which require more frequent nasopharyngeal suctioning, and in turn, could have contributed to the increased risk of CoNS colonisation and/ or sepsis. A third limitation was our choice of cut-off period (i.e. 48 hours of exposure to various potential risk factors), which was based on our previous bedside observation, since no previous study (animal or clinical) has shown scientifically the causal relationship between a minimal duration of exposure and colonisation/sepsis.

The findings of the present study are not without implications. As such, a multicentre study with a large sample size should be carried out to validate our findings. If the findings of such a study are similar to ours, the policy and procedures for nasopharyngeal and/or oral suctioning in NICUs should be reviewed.

\section{REFERENCES}

1. Kloos WE, Bannerman TL. Update on clinical significance of coagulasenegative staphylococci. Clin Microbiol Rev 1994; 7:117-40.

2. Hira V, Sluijter M, Estevão S, et al. Clinical and molecular epidemiologic characteristics of coagulase-negative staphylococcal bloodstream infections in intensive care neonates. Pediatr Infect Dis J 2007; 26:607-12.

3. Gray JE, Richardson DK, McCormick MC, Goldmann DA. Coagulasenegative staphylococcal bacteremia among very low birth weight infants: relation to admission illness severity, resource use, and outcome. Pediatrics 1995; 95:225-30.

4. Stoll BJ, Gordon T, Korones SB, et al. Late-onset sepsis in very low birth weight neonates: a report from the National Institute of Child Health and Human Development Neonatal Research Network. J Pediatr 1996; 129:63-71.

5. Gaynes RP, Edwards JR, Jarvis WR, et al. Nosocomial infections among neonates in high-risk nurseries in the United States. National Nosocomial Infections Surveillance system. Pediatrics 1996; 98:357-61.

6. Fanaroff AA, Korones SB, Wright LL, et al. Incidence, presenting features, risk factors and significance of late onset septicemia in very low birth weight infants. The National Institute of Child Health and Human Development Neonatal Research Network. Pediatr Infect Dis J 1998; 17:593-8.

7. Isaacs D; Australasian Study Group For Neonatal Infections. A ten year multicentre study of coagulase negative staphylococcal infections in Australasian neonatal units. Arch Dis Child Fetal Neonatal Ed 2003; 88:F89-93.

8. Stoll BJ, Hansen N, Fanaroff AA, et al. Late-onset sepsis in very low birth weight neonates: the experience of the NICHD Neonatal Research Network. Pediatrics 2002; 110:285-91

9. Costa SF, Miceli MH, Anaissie EJ. Mucosa or skin as source of coagulasenegative staphylococcal bacteraemia? Lancet Infect Dis 2004; 4:278-86.

10. Vermont CL, Hartwig NG, Fleer A, et al. Persistence of clones of coagulasenegative staphylococci among premature neonates in neonatal intensive care units: two-center study of bacterial genotyping and patient risk factors. J Clin Microbiol 1998; 36:2485-90.

11. Freeman J, Goldmann DA, Smith NE, et al. Association of intravenous lipid emulsion and coagulase-negative staphylococcal bacteremia in neonatal intensive care units. N Engl J Med 1990; 323:301-8.

12. Low DE, Schmidt BK, Kirpalani HM, et al. An endemic strain of Staphylococcus haemolyticus colonizing and causing bacteremia in neonatal intensive care unit patients. Pediatrics 1992; 89:696-700.

13. Eastick K, Leeming JP, Bennett D, Millar MR. Reservoirs of coagulase negative staphylococci in preterm infants. Arch Dis Child Fetal Neonatal Ed 1996; 74: F99-104.

14. Bétrémieux P, Donnio PY, Pladys P. Use of ribotyping to investigate tracheal colonization by Staphylococcus epidermidis as a source of bacteremia in ventilated newborns. Eur J Clin Microbiol Infect Dis 1995; 14:342-6.

15. Björkqvist M, Liljedahl M, Zimmermann J, Schollin J, Söderquist B. Colonization pattern of coagulase-negative staphylococci in preterm neonates and the relation to bacteremia. Eur J Clin Microbiol Infect Dis 2010; 29:1085-93.

16. Rønnestad A, Abrahamsen TG, Medbø S, et al. Late-onset septicemia in a Norwegian national cohort of extremely premature infants receiving very early full human milk feeding. Pediatrics 2005; 115:e269-76. 